\title{
UNITARY BORDISM OF ABELIAN GROUPS
}

\section{ERICH OSSA ${ }^{1}$}

ABstract. It is shown that for a finite abelian group $G$ the bordism group of unitary $G$-manifolds is a free $U_{*}$-module on even dimensional generators.

Introduction. In this note we study from the bordism point of view actions of a finite abelian group $G$ on unitary manifolds such that the action commutes with the stable complex structure on the tangent bundle. The corresponding bordism group $U_{*}(G)$ is a graded module over the complex bordism ring $U_{*}$.

In [4] Stong had shown that for a finite $p$-primary group $G$ the module $U_{*}(G)$ is free on even dimensional generators. Landweber [3] proved the same result for finite cyclic $G$.

Using Stong's result we give a simple proof of the following.

THEOREM 1. Let $G$ be a finite abelian group. Then $U_{*}(G)$ is a free $U_{*}$ module on even dimensional generators.

In [2] it was shown how such results for finite groups imply similar ones for higher dimensional groups. In fact, from the knowledge of Theorem 1 the methods of [2] give immediately

THEOREM 2. Let $G$ be a compact abelian Lie group and let $U_{*}^{(j)}(G)$ be the bordism module of actions such that each isotropy subgroup has codimension at least $j$. Then $U_{*}^{(j)}(G)$ is a free $U_{*}$-module on generators in dimension congruent to $j$ modulo 2.

In [2] this theorem was proved for a monogenic group $G$, using Landweber's result instead of Theorem 1.

Throughout the paper $G$ will be a finite abelian group. The order of $G$ is denoted by $|G|$.

1. Preliminaries. We shall have to consider also bordism modules of $G$-vectorbundles. The most general bordism group arising in this connection is defined as follows:

Received by the editors September 1, 1971.

AMS 1970 subject classifications. Primary 57D85.

$K e y$ words and phrases. Unitary $G$-manifolds, equivariant bordism group.

${ }^{1}$ Supported in part by National Science Foundation grant GP $7952 \mathrm{X} 2$.

(c) American Mathematical Society 1972 
Let $k_{1}, \cdots, k_{m}$ be nonnegative integers and let $F, F_{1}, \cdots, F_{m}$ be families of subgroups of $G$. Consider complex $G$-vectorbundles $E_{1}, \cdots, E_{m}$ over closed unitary $G$-manifolds $M$ (in the sense of the Introduction) with the following properties:

(i) All isotropy groups in $M$ are elements of $F$,

(ii) $E_{i}$ is of dimension $k_{i}$ and all isotropy groups in the sphere bundle of $E_{i}$ are elements of $F_{i}$.

The corresponding bordism group of such objects $\left(M, E_{1}, \cdots, E_{m}\right)$ will be denoted by $U_{*}\left(G ; F ;\left(k_{1}, F_{1}\right), \cdots,\left(k_{m}, F_{m}\right)\right)$. If any of the families $F, F_{1}, \cdots, F_{m}$ is the family of all subgroups of $G$ we delete it from the notation; moreover, we will simply write $(k)$ instead of $k_{1}, \cdots$, $k_{m}$.

We shall actually prove

THEOREM 1'. Let $G$ be a finite abelian group. Then $U_{*}(G ;(k))$ is a free $U_{*}$-module on even dimensionai generators.

This induces a similar generalization of Theorem 2.

Our basic tool is the well-known exact sequence introduced by Conner [1]. Let $F$ be as above and let $H$ be an element of $F$. Define $F_{H}=$ $\left\{H^{\prime} \in F \mid H \subset H^{\prime}\right\}$ and $F^{\prime}=F-F_{H}$. Then the following sequence of $U_{*^{-}}$ modules is exact:

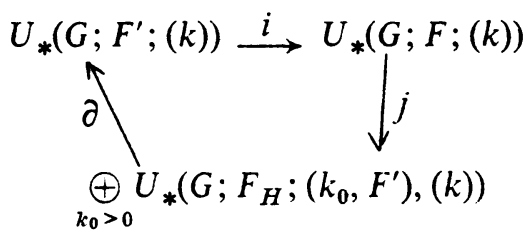

Here $i$ is the obvious forgetful map,

$$
j\left(M, E_{1}, \cdots, E_{m}\right)=\sum_{i}\left(M_{i}^{H}, v_{i}, E_{1}\left|M_{i}^{H}, \cdots, E_{m}\right| M_{i}^{H}\right)
$$

where $M_{i}^{H}$ is a component of the fixed point set of $H$ in $M$ and $v_{i}$ its normal bundle, and finally $\partial\left(M, E_{0}, E_{1}, \cdots, E_{m}\right)=\left(S, \pi^{*} E_{1}, \cdots, \pi^{*} E_{m}\right)$ where $\pi: S \rightarrow M$ is the projection of the sphere bundle $S$ of $E_{0}$. The exactness follows from obvious geometric arguments.

For an element $\left(M, E_{0}, E_{1}, \cdots, E_{m}\right)$ in $U_{*}\left(G ; F_{H} ;\left(k_{0}, F^{\prime}\right),(k)\right)$ the action of $H$ is just given by representations of $H$. It is possible to describe the $G$-action by means of these representations and a $G / H$-action. Then in many cases $U_{*}\left(G ; F_{H} ;\left(k_{0}, F^{\prime}\right),(k)\right)$ is isomorphic to a direct sum of bordism modules $U_{*}(G / H ; \tilde{F} ;(\tilde{k})$ ) (see for instance [2, proof of Lemma 1] where a similar situation is studied in detai1). Later on we shall use such isomorphisms without further notice. 
2. Proof of Theorem $\mathbf{1}^{\prime}$. Write the finite abelian group $G$ in the form $G=K \times L$ where the orders $|K|$ and $|L|$ are relatively prime. Let $d=|K|$ and let $R_{d}$ be the ring $R_{d}=Z[1 / d] \subset Q$.

Proposition 2. Let $F$ be a family of subgroups of $L$ and let $f$ : $U_{*}(K \times L ; F ;(k)) \rightarrow U_{*}(L ; F ;(k))$ be defined by forgetting the action of $K$. Then $f \otimes \mathrm{id}: U_{*}(K \times L ; F ;(k)) \otimes R_{d} \rightarrow U_{*}(L ; F ;(k)) \otimes R_{d}$ is an isomorphism.

REMARK. The inverse isomorphism is then given by multiplication with $[K] \otimes 1 / d$ where $[K]$ is the bordism class of the free $K$-manifold $K$.

Proof of Proposition 2. We proceed by induction over $L$ and the family $F$.

If $F=\{\{1\}\}$ contains only the trivial group we have $U_{*}(K \times L ; F ;(k)) \cong$ $U_{*}\left(B K \times B L \times \prod B U\left(k_{i}\right)\right)$ where the right-hand side is the usual bordism group of the classifying space $B\left(K \times L \times \prod U\left(k_{i}\right)\right)$. But since $\tilde{H}_{*}\left(B K ; R_{d}\right)=$ 0 it follows that

$$
\begin{aligned}
U_{*}\left(B K \times B L \times \prod B U\left(k_{i}\right)\right) \otimes R_{d} & \cong U_{*}\left(B L \times \prod B U\left(k_{i}\right)\right) \otimes R_{d} \\
& \cong U_{*}(L ; F ;(k)) \otimes R_{d}
\end{aligned}
$$

and this isomorphism is given by $x \mapsto f(x) \otimes 1 / d$. Hence $f \otimes$ id is an isomorphism for $F=\{\{1\}\}$.

Now let $F$ be arbitrary and $H$ a maximal element of $F$. Set $F^{\prime}=F-\{H\}$. Then one has the following commutative diagram of exact sequences:

$\partial$

$\longrightarrow U_{*}\left(K \times L ; F^{\prime} ;(k)\right) \stackrel{i}{\longrightarrow} U_{*}(K \times L ; F ;(k)) \stackrel{j}{\longrightarrow} \oplus U_{*}(K \times L / H ;\{\{1\}\} ;(\tilde{k}))$

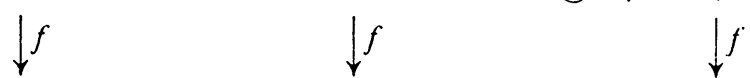

$\left[\quad U_{*}\left(L ; F^{\prime} ;((k)) \stackrel{i}{\longrightarrow} U_{*}(L ; F,(k)) \stackrel{j}{\longrightarrow} \oplus\left(U_{*}(L / H ;\{\{1\}\} ;(\tilde{k}))-\right]\right.\right.$

Tensoring with $R_{d}$, the ind:ction hypothesis, and the 5-lemma give then the desired result.

Proposition 3. Assume that $U_{*}(L ;(k)) \otimes R_{d}$ is a free $U_{*} \otimes R_{d}$-module on even dimensional generators for all $(k)$. Let $F$ be a family of subgroups of $K$, $\Phi$ the family of all subgroups of $L$ and $F \times \Phi=\left\{H \times H^{\prime} \mid H \in F, H^{\prime} \in \phi\right\}$. Then $U_{*}(K \times L ; F \times \Phi ;(k)) \otimes R_{d}$ is a free $U_{*} \otimes R_{d}$-module on even dimensional generators.

Pruof. We proceed by induction over $K$ and $F$. The case $F=\{\{1\}\}$ is just Proposition 2 together with the assumption. 
Let $H$ be a maximal element of $F$ and $F^{\prime}=F-\{H\}$. Then we have the exact sequence

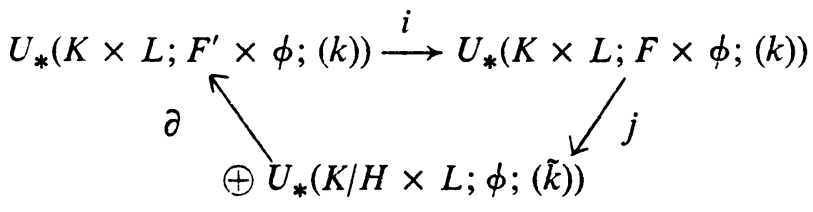

Tensoring with $R_{d}$, applying the induction hypotheses, and separating even and odd dimensions ( $i$ and $j$ preserve dimensions, $\partial$ lowers dimensions by 1 ) yields the result.

COROLlaRY 4. If $U_{*}(L ;(k)) \otimes R_{d}$ is a free $U_{*} \otimes R_{d}$-module on even dimensional generators for all $(k)$, then the same statement holds for $U_{*}(K \times L ;(k)) \otimes R_{d}$.

Proof. Since $|K|$ and $|L|$ are relatively prime, any subgroup of $K \times L$ is a direct product of subgroups of $K$ and $L$.

Now we are able to prove Theorem 1' by induction over the number of primary factors of $G$. For $G$ p-primary the result was proved in [4]. For arbitrary $G$ the induction hypothesis and Corollary 4 show that for any integer $d>1$ dividing $|G|$ the $U_{*} \otimes R_{d}$-module $U_{*}(G ;(k)) \otimes R_{d}$ is free on even dimensional generators. The assertion follows now from the trivial:

LEMMA 5. Let $M$ be a graded $U_{*}$-module of finite type (that is $M / \oplus_{n \geqq N} M^{n}$ is finitely generated for any $\left.N\right)$. Let $d_{1}, d_{2}$ be relatively prime and assume that $M \otimes R_{d_{i}}$ is a free $U_{*} \otimes R_{d_{i}}$-module on even dimensional generators. Then $M$ is a free $U_{*}$-module on even dimensional generators.

\section{REFERENCES}

1. P. E. Conner, A bordism theory for actions of an abelian group, Bull. Amer. Math. Soc. 69 (1963), 244-247. MR 26 \#4353.

2. G. Hamrick and E. Ossa, Unitary bordism of monogenic groups and isometries, Proc. Conference on Transformation Groups, Amherst, 1971 (to appear).

3. P. S. Landweber, Equivariant bordism and cyclic groups, Proc. Amer. Math. Soc. 31 (1972), 564-570.

4. R. E. Stong, Complex and oriented equivariant bordism, Topology of Manifolds, J. C. Cantrell and C. H. Edwards, Jr. (Editors), Markham, Chicago, Ill., 1970, pp. 291-316.

Department of Mathematics, Rheinische Friedrich-Wilhelms-Universität zU Bonn, Bonn, federal Republic of Germany

Institute for Advanced Study, Princeton, New Jersey 08540 